\title{
New transport experiments in strange metals
}

\section{Quantum-critical continuum in magic-angle twisted bilayer graphene}

Authors: Alexandre Jaoui, Ipsita Das, Giorgio Di Battista, Jaime Díez-Mérida, Xiaobo

Lu, Kenji Watanabe, Takashi Taniguchi, Hiroaki Ishizuka, Leonid Levitov, Dmitri

K.Efetov

https://arxiv.org/abs/2108.07753

2. Incoherent transport across the strange metal regime of highly overdoped cuprates Authors: J. Ayres, M. Berben, M. Culo, Y. -T. Hsu, E. van Heumen, Y. Huang, J. Zaanen, T. Kondo, T. Takeuchi, J. R. Cooper, C. Putzke, S. Friedemann, A. Carrington, N. E. Hussey

Nature, vol. 595, 661-666 (2021)

\section{Linear-in temperature resistivity from an isotropic Planckian scattering rate} Authors: G. Grissonnanche, Y. Fang, A. Legros, S. Verret, F. Laliberté, C. Collignon, J. Zhou, D. Graf, P. Goddard, L. Taillefer, B. J. Ramshaw Nature 595, 667-672 (2021)

\section{Recommended with a Commentary by T. Senthil, Massachusetts Institute of Technology}

In diverse correlated electron metals, departures from Landau's Fermi liquid theory happen down to temperatures much lower than the natural energy scales characterizing the system. Such metals are collectively dubbed 'strange metals' to reflect our lack of a general theoretical understanding. A prominent example is in the normal state of high Tc cuprates near optimal doping. Famously the electrical resistivity is linear in temperature over a wide range of temperature. Suppressing the superconductivity with a magnetic field reveals that the linear resistivity persists down essentially to the zero temperature limit. Similar linear resistivity is seen in a number of different metals.

The featured paper by Jaoui et al studies transport in Magic Angle Twisted Bilayer Graphene (MATBG). The slew of fascinating phenomena discovered in this system includes linear-in-temperature resistivity[1,2] over a range of dopings and temperature. The linear resistivity was seen to extend to rather low temperatures near the moire lattice fillings at which correlated insulators are seen. Nevertheless it has been suggested[2,3] that electron-phonon scattering could account for the linear resistivity, albeit with a mysterious lowering of the BlochGruneisen temperature. The paper by Jaoui et al studies MATBG devices with a proximate screening gate (the fate of correlation driven insulators and superconductivity in such devices 
had been studied previously[4] by the same group). Linear-T resistivity is found over 3 decades in temperature, and goes down to about $40 \mathrm{mK}$ in a range of moire lattice filling around -2 . For comparison, the estimated Bloch-Gruneisen temperature scale is about a few $\mathrm{K}$. The challenge for theories based on electron-phonon scattering to account for the linear resistivity has thus become a lot steeper. Further the linear resistivity disappears at dopings near the band edges or in devices further away from the magic twist angle, pointing to the importance of correlation effects.

A final interesting observation is that in an out-of-plane magnetic field, the magnetoresistance is linear in the regimes in which linear-in-T resistivity is found. A linear magnetoresistance has been reported with growing frequency in a variety of strange metals, for instance the iron pnictides[5], various cuprates[6,7], and twisted transition metal dichalcogenides[8]. Thus its observation in MATBG raises hopes for a common phenomenology despite the well-known microscopic differences with conventional correlated materials.

The second featured paper by Ayres, Hussey et al also studies magnetotransport but in the venerated hole-doped cuprate materials. A striking linear magnetoresistance down to low $\mathrm{T}$ in the strange metal regime was previously reported in cuprate thin films in Ref. [6] when superconductivity was suppressed by the use of high (up to $80 \mathrm{~T}$ ) fields. Ayres et al study the nature of the metallic phase in two families of overdoped cuprates. In 2009, Hussey's group showed[9] that an extrapolation to zero field of magnetic field dependent transport data in overdoped $\mathrm{La}_{2-x} \mathrm{Sr}_{x} \mathrm{CuO}_{4}$ yielded (at $\mathrm{T} \rightarrow 0$ ) a linear-T resistivity all the way up to the edge of the superconducting dome. This surprising claim called into question the conventional picture of a single quantum critical doping associated with the strange metal. However the procedure to extrapolate to zero magnetic field used in the 2009 paper left room for some concern on whether what was plotted was truly indicative of the resistivity of an underlying normal metallic state at low T.

In their new paper, Ayres, Hussey et al find a number of potentially significant results. First the magnetoresistance is seen to be linear up to the edge of the superconducting dome. Further an extrapolation of the linear magnetoresistance to zero field demonstrates with more confidence the linear-T resistivity through out this doping range. Third the linear magnetoresistance does not fit a simple scaling form (for instance the quadrature scaling $\sqrt{\left(\alpha k_{B} T\right)^{2}+\left(\gamma \mu_{0} \mu_{B} H\right)^{2}}$ suggested in Ref.[5] for $\left.\mathrm{BaFe}_{2}\left(A s_{1-x} P_{x}\right)_{2}\right)$; rather the data is fit to a more complex form $\rho(T, H)=\mathscr{F}(T)+\sqrt{\left(\alpha k_{B} T\right)^{2}+\left(\gamma \mu_{0} \mu_{B} H\right)^{2}}$.

The similarity with the observation of linear-T resistivity, and linear magnetoresistance in a range of dopings in MATBG should be noted. There are also some differences: the detailed T and $\mathrm{H}$ dependence of the resistivity in MATBG is not of the forms discussed in the previous paragraph. It will be important for future experiments in both these systems to clarify the nature of this extended doping regime with potential strange metal transport. For instance the possibility 
of quantum oscillations and their temperature dependence can be examined and will provide useful information.

The transport data on many metals has been used to suggest[10,11,12] some sort of fundamental bound (dubbed the 'Planckian bound') on the temperature dependent electronic scattering rate $\Gamma=c \frac{k_{B} T}{\hbar}$ (with $\mathrm{c}$ a dimensionless constant of order 1). In common with previous work $[13,1]$ both Jaoui et al and Ayres et al associate such a scattering rate with the linear-T resistivity found in the strange metal regime.

In the featured paper by Grissonanche, Ramshaw et al, the scattering rate is extracted through a Boltzmann analysis of Angle Dependent Magneto-Resistance (ADMR) data. ADMR measures oscillations in the c-axis resistivity of a layered material as the orientation of an external magnetic field is varied. In a Fermi liquid metal, the electronic quasiparticles will experience periodically varying Fermi velocities and scattering rates as they move around the Fermi surface due to the Lorentz force, and this leads to resistance oscillations. Detailed modeling of the ADMR data can help determine the shape of the Fermi surface and extract (angle dependent) scattering rates. Grissonanche et al. study ADMR in a cuprate strange metal (Nd-doped LSCO) at a doping $p=0.24$ close to a putative pseudogap quantum critical point down to $T \approx 6 K$. At this doping level it is known[14] that the ab-plane resistivity is linear-in-T down to about $1 \mathrm{~K}$. The detailed modeling of the ADMR data shows that the scattering rate is the sum of an isotropic (angle independent) component and an anisotropic one that is large at the antinodes and small at the nodes. The anisotropic component is T-independent and it is natural to attribute to elastic scattering. Interestingly the isotropic component follows the temperature dependence of the suggested Planckian limit with $c \approx 1.2 \pm 0.4$.

It is tempting to draw the conclusion that a saturation of the Planckian scattering limit is a necessary feature of strange metals with linear resistivity. Caution is however needed: at very low-T the specific heat coefficient $\gamma$ of the same cuprate is known[15] to have a $\ln (1 / T)$ divergence, signaling a possible divergence of the quasiparticle effective mass. The resistivity however stays linear in this regime. It is not a priori clear that the concept of a 'scattering rate' is well-defined in the presence of such a divergent effective mass. Indeed a naive application of the Drude formula $\rho(T)=\rho(0)+\frac{m^{*} \Gamma_{\text {inel }}}{n e^{2}}$ to define an inelastic scattering rate $\Gamma_{\text {inel }}$ yields a sub-Planckian $\frac{T}{\ln (1 / T)}$ form which clearly does not saturate the suggested Planckian limit despite the linear-T resistivity.

There is, as far as I am aware, no theoretically precise and general statement of a Planckian bound for electrical transport (leave alone a proof). Nevertheless the appearance of something resembling a Planckian scattering rate in a number of strange metals suggests that a precise statement may exist and may be a good target for future theory. Finally the authors suggest that the anisotropic scattering found may play a role in understanding the linear magnetoresistance. 


\section{References}

[1] Yuan Cao, Debanjan Chowdhury, Daniel Rodan-Legrain, Oriol Rubies-Bigordà, Kenji Watanabe, Takashi Taniguchi, T. Senthil, Pablo Jarillo-Herrero, Phys. Rev. Lett. 124, 076801 (2020).

[2] Hryhoriy Polshyn, Matthew Yankowitz, Shaowen Chen, Yuxuan Zhang, K Watanabe, T Taniguchi, Cory R Dean, Andrea F Young, Nature Physics, 15, 1011-1016 (2019).

[3]. F Wu, E Hwang, S Das Sarma, Physical Review B 99 (16), 165112 (2019).

[4]. P. Stepanov, I. Das, X. Lu, A. Fahimniya, K. Watanabe, T. Taniguchi, F.H.L. Koppens, K. Lischner, L. Levitov, D.K. Efetov, Nature 583, 375-378 (2020).

[5]. Ian M Hayes, Ross D McDonald, Nicholas P Breznay, Toni Helm, Philip JW Moll, Mark Wartenbe, Arkady Shekhter, James G Analytis, Nature Physics 12 (10), 916-919 (2016).

[6]. Paula Giraldo-Gallo et al, Science 361 (6401), 479-481 (2018).

[7]. Richard L. Greene, Pampa R. Mandal, Nicholas R. Poniatowski, Tarapada Sarkar, Annu. Rev. Condens. Matter Phys 11213 (2020).

[8]. Augusto Ghiotto et al, Nature 597 (2021) 345-349 (2021).

[9]. RA Cooper et al, Science 323 (5914), 603-607 (2009).

[10]. J. Zaanen, Nature Physics 12 (10), 916-919 (2004).

[11]. JAN Bruin, H Sakai, RS Perry, AP Mackenzie, Science 339 (6121), 804-807

[12]. Sean Hartnoll, Andrew Lucas, Subir Sachdev, MIT Press (2018).

[13]. A Legros et al, Nature Physics 15 (2), 142-147 (2018).

[14]. R Daou et al, Nature 463 (7280), 519-522 (2010).

[15]. B Michon et al , Nature 567 (7747), 218-222 (2019). 